\title{
Bisdemethoxycurcumin suppresses migration and invasion of highly metastatic 95D lung cancer cells by regulating E-cadherin and vimentin expression, and inducing autophagy
}

\author{
JINHONG XU, HEPING YANG, XIANGDONG ZHOU, HAIJING WANG, LIANG GONG and CHUNLAN TANG
}

Department of Respiratory Medicine, Southwest Hospital, The Third Military Medical University, Chongqing 400038, P.R. China

Received October 15, 2014; Accepted June 30, 2015

DOI: $10.3892 / \mathrm{mmr} .2015 .4356$

\begin{abstract}
Curcumin is an active component of the medicinal plant turmeric, which has been reported to have anti-metastatic activities and induce autophagy in numerous cancer types. Bisdemethoxycurcumin (BDMC), one of the major active curcumin derivatives present in turmeric, was previously shown to trigger autophagy in highly metastatic large-cell lung cancer 95D cells. However, the effects of the induction of autophagy by BDMC on the invasion and migration of 95D cells has remained elusive. Therefore, the present study investigated the effects of BDMC on the invasion and migration of highly metastatic large-cell lung cancer 95D cells. Meanwhile we observed the effect of autophagy induced by BDMC on the migration and invasion in 95D cells. Transwell assays showed that BDMC exerted an inhibitory effect on the migration and invasion of 95D cells. Furthermore, the expression of vimentin was downregulated, while E-cadherin expression was upregulated in 95D cells treated with BDMC. In addition, blockage of autophagy through Beclin1-targeted small interfering RNA attenuated the inhibition of BDMC on 95D-cell migration and invasion. These findings provided direct evidence that BDMC inhibits 95D-cell migration and invasion. Furthermore, the inhibition of 95D-cell migration and invasion was associated with the downregulation of vimentin expression and the upregulation of E-cadherin expression. Autophagy was involved in the anti-cancer effects of BDMC on $95 \mathrm{D}$ cells. The present study provided novel insight into the underlying mechanisms of the anti-cancer effect of BDMC.
\end{abstract}

Correspondence to: Dr Heping Yang, Department of Respiratory Medicine, Southwest Hospital, The Third Military Medical University, 30 Gaotanyan Street, Sha-Ping-Ba, Chongqing 400038, P.R. China E-mail: heping_yang6@163.com

Key words: bisdemethoxycurcumin, invasion, migration, autophagy, E-cadherin, vimentin

\section{Introduction}

Lung cancer is the leading cause of cancer-associated mortality worldwide. It was estimated that 226,160 novel cases and 160,340 mortalities from cancer occurred in the United States in 2012 (1). Non-small cell lung cancer (NSCLC) accounts for nearly $80-85 \%$ of all lung cancer types. Approximately 40-50\% of patients with NSCLC present at an advanced stage, with metastatic or locally advanced disease (2). Despite all recent improvements in chemotherapy strategies and surgeries, the five-year survival rate has remained $<15 \%$ due to the emergence of serious adverse effects, cancer recurrence and resistance to conventional chemotherapy. Furthermore, among these patients with tumor recurrence, $>70 \%$ present with distant metastasis (3). Cancer cell invasion and migration are critical processes determining the lethality of a malignancy, and $>90 \%$ of cancer mortalities have been attributed to the metastatic spread of the disease rather than to the primary tumor. Tumor metastasis is the main obstacle on the treatment of lung cancer. Therefore, novel targeted therapeutic agents with high efficacy and low toxicity for inhibiting metastasis are urgently required.

Curcuminoids are the major active components of turmeric with significant derivatives including curcumin (Cur), demethoxycurcumin (DMC) and bisdemethoxycurcumin (BDMC). Curcumin possesses a wide variety of biological activities, including anti-proliferative, anti-oxidant, anti-inflammatory, anti-angiogenic and anti-tumor effects $(4,5)$. It has been shown that curcumin exerts marked anti-cancer effects through modulating the expression of a variety of molecules involved in cancer progression (6). Curcumin has been demonstrated to suppress lung cancer cell invasion and metastasis through multiple signaling pathways, including DnaJ-like heat shock protein 40 (HLJ1) (7), matrix metalloproteinase-2, metalloproteinase-9, vascular endothelial growth factor (8) and Janus kinase-signal transducer and activator of transcription 3 signaling pathway (9). However, the stability of natural curcumin is poor and it can be easily degraded in vivo. Therefore, it is necessary to use other stable curcuminoids. BDMC is thought to be more stable than the other two curcuminoids (10). Previous studies have shown that BDMC has the highest anti-metastatic potency in HT1080 human fibrosacroma among the three curcuminoids (11). However, 
the underlying molecular mechanisms of BDMC on tumor invasion and migration have largely remained to be identified.

Autophagy is known to be a double-edged sword in tumorigenesis and cancer development. Manipulation of autophagy has been reported to have the potential to enhance the anti-cancer effects of chemotherapeutics in the clinic (12). It has been reported that CLEFMA, a synthetic analog of curcumin, induced autophagy in lung adenocarcinoma H441 cells (13). Furthermore, curcumin induced autophagy in A549 cells (14). However, little is known about the association between autophagy induced by curcumin and the migratory and invasive potential of NSCLC cells.

The present study investigated the effects of BDMC on the proliferation, migration and invasion of 95D cells and explored the underlying molecular mechanisms by detecting vimentin and E-cadherin expression. In addition, the role of autophagy in the invasion and migration of 95D cells was investigated.

\section{Materials and methods}

Reagents and antibodies. BDMC was purchased from Sigma-Aldrich (St. Louis, MO, USA). BDMC ( $>98 \%$ pure) was dissolved in dimethyl sulfoxide (DMSO; Sigma-Aldrich) to prepare a $100-\mathrm{mM}$ stock solution, which was diluted with medium to the required concentrations. The final concentration of DMSO was not more than $0.01 \%$. Polyclonal anti-GAPDH antibody (cat. no. G9545) was purchased from Sigma-Aldrich and rabbit polyclonal antibody against vimentin (cat. no. sc-5565) was procured from Santa Cruz Biotechnology, Inc. (Dallas, TX, USA). Rabbit monolconal antibody against E-cadherin (cat. no. 3195) was from Cell Signaling Technology (Beverly, MA, USA). Goat anti-rabbit immunoglobulin $\mathrm{G}$ horseradish peroxidase-conjugated secondary antibody (cat. no. ZDR-5306) was from Zhongshan Golden Bridge Biotechnology (Beijing, China).

Cell lines, cell culture and transfection. The highly metastatic large-cell lung cancer 95D cell line was obtained from the Chinese Academy of Science Cell Bank (Shanghai, China). Cells were cultured in RPMI 1640 medium (GE Healthcare Life Sciences, Logan, UT, USA) supplemented with $10 \%$ (v/v) fetal bovine serum (FBS; Gibco-BRL, Invitrogen Life Technologies, Carlsbad, CA, USA) in a humidified atmosphere at $37^{\circ} \mathrm{C}$ with $5 \% \mathrm{CO}_{2}$. Transient transfection was performed with green fluorescence protein-Beclin 1 plasmid using Lipofectamine 2000 (Invitrogen Life Technologies) according to the manufacturer's instructions.

Cytotoxicity and cell proliferation assay. The cytotoxicity and proliferation of $95 \mathrm{D}$ cells after exposure to BDMC was measured using the cell counting kit 8 (CCK-8) assay kit (Beyotime Institute of Biotechnology, Haimen, China). Briefly, $4 \times 10^{3}$ cells in the logarithmic growth phase suspended in $100 \mu 1$ complete medium were seeded into each well of a 96-well flat-bottomed plate (Nest Biotech Co. Ltd., Wuxi, China) and incubated at $37^{\circ} \mathrm{C}$ in a humidified $5 \%-\mathrm{CO}_{2}$ atmosphere overnight. The cells were then treated with various concentrations of $\operatorname{BDMC}(0,5,10,20,40$ or $80 \mu \mathrm{M})$ for $24 \mathrm{~h}$. Subsequently, $10 \mu \mathrm{l}$ CCK8 reagent was added to each well containing $100 \mu \mathrm{l}$ of culture medium, followed by incubation for $1 \mathrm{~h}$ at $37^{\circ} \mathrm{C}$.

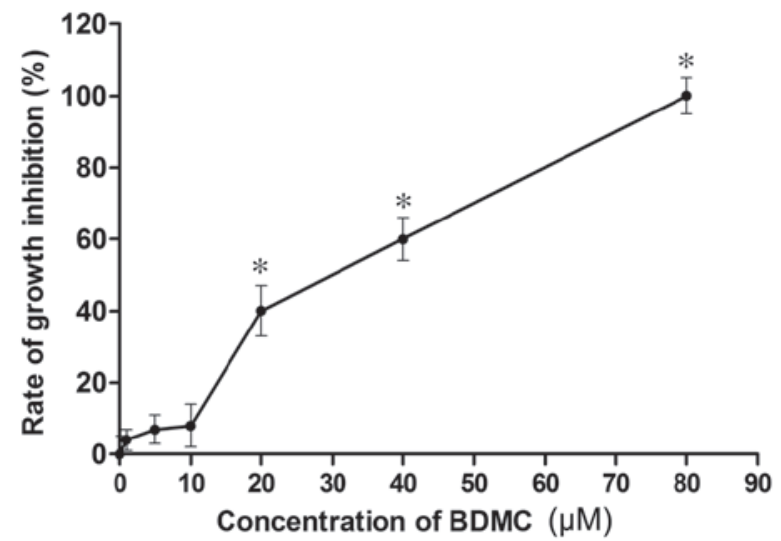

Figure 1. Cell counting kit 8 assay showing the effect of BDMC on the proliferation of 95D cells. The 95D cells were treated with up to $0-80 \mu \mathrm{M}$ BDMC for $24 \mathrm{~h}$. Values are expressed as the mean \pm standard deviation $(n=3)$. ${ }^{*} \mathrm{P}<0.05$ vs. untreated cells. BDMC, bisdemethoxycurcumin.

The optical density (OD) at $450 \mathrm{~nm}$ was measured using a microplate spectrophotometer (Bio-Rad Laboratories, Inc., Hercules, CA, USA). All of the experiments were performed three times.

In vitro invasion assay. A total of $1 \times 10^{5}$ cells $95 \mathrm{D}$ cells in the logarithmic growth phase in $0.5 \mathrm{ml}$ serum-free RPMI 1640 medium were seeded into $8 \mu \mathrm{m}$-pore polycarbonate membrane chamber inserts in a Transwell plate (Corning-Costar, Corning, NY, USA) coated with Matrigel (BD Biosciences, Franklin Lakes, NJ, USA). $600 \mu \mathrm{l}$ RPMI 1640 containing 20\% FBS was added to the lower chamber. After the cells were incubated for 12-24 h, cells on the upper surface of the insert were removed by wiping with a cotton swab. Cells that transgressed through the membrane to the bottom surface of the insert were fixed in $100 \%$ methanol for $10 \mathrm{~min}$, stained in $0.5 \%$ crystal violet (Sigma-Aldrich) for $10 \mathrm{~min}$, rinsed with phosphate-buffered saline and then subjected to microscopic inspection (magnification, x100; Olympus CX22, Olympus Corporation, Tokyo, Japan). The invasion was quantified by counting five fields per membrane. Values are and expressed as the average of three independent experiments.

Cell motility assay. Cell migration assays were performed using Boyden chambers containing polycarbonate membrane inserts ( $8 \mu \mathrm{m}$ pore size; Corning Costar) Briefly, $1 \times 10^{4}$ cells in $0.1 \mathrm{ml}$ serum-free RPMI-1640 medium were seeded into the upper chamber. $600 \mu 1 \mathrm{RPMI}-1640$ containing $20 \%$ FBS was added to the lower chamber as a chemoattractant. After the cells were incubated for 6,12 or $24 \mathrm{~h}$ at $37^{\circ} \mathrm{C}$ in a $5 \%-\mathrm{CO}_{2}$ incubator, the number of cells that had migrated to the lower surface was counted. The experiments were repeated a minimum of three times.

RNA interference of Beclin 1. 95D cells were seeded in 24 -well plates $\left(1.5 \times 10^{5}\right.$ cells/well $)$ and incubated overnight. A negative control random small interfering RNA (NC-siRNA) and Beclin1-targeted siRNA (1 $\mu \mathrm{g} /$ well) were purchased from Genepharma (Shanghai, China). NC-siRNA and Beclin1-siRNA were transfected using Lipofectamine 2000 according to the manufacturer's instructions. 
A

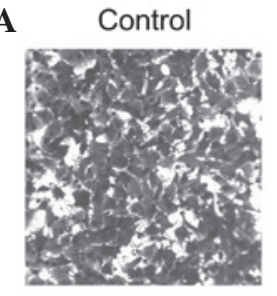

BDMC $(5 \mu \mathrm{M})$
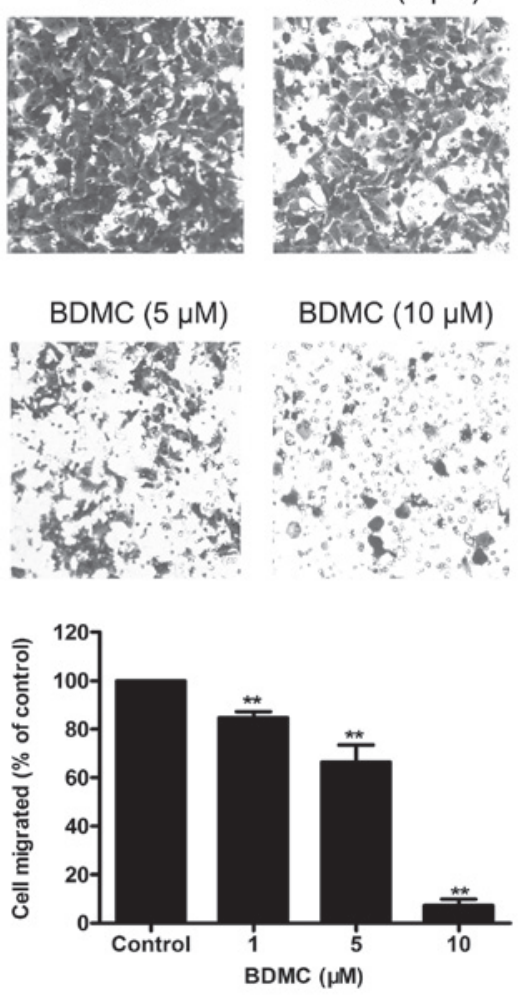

$\operatorname{BDMC}(1 \mu \mathrm{M}) \quad$ B
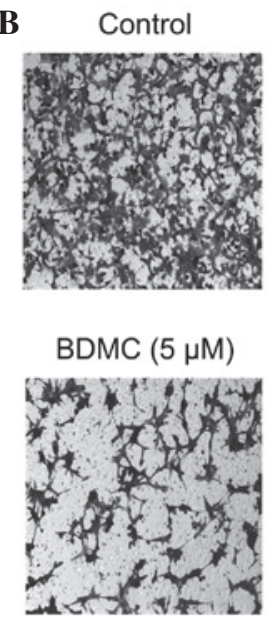

$\operatorname{BDMC}(1 \mu \mathrm{M})$

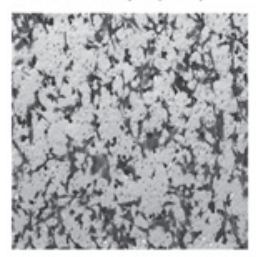

$\operatorname{BDMC}(10 \mu \mathrm{M})$
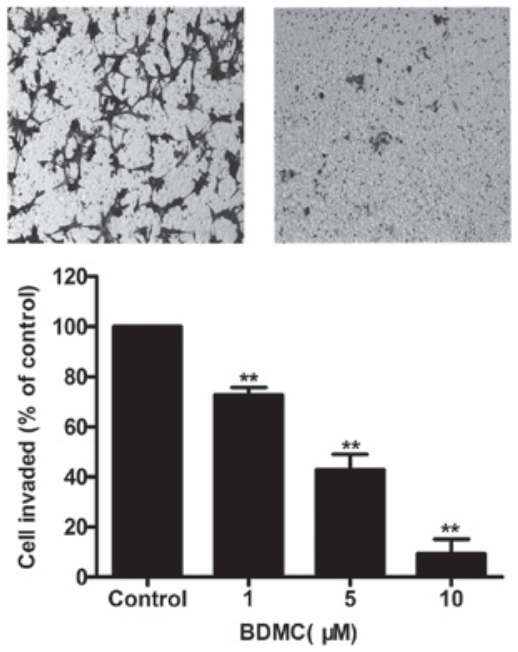

Figure 2. BDMC inhibits (A) the migration and (B) the invasion of 95D cells. Boyden chamber/Transwell assays were performed to determine the effects of $\operatorname{BDMC}(1,5$ and $10 \mu \mathrm{M} ; 24 \mathrm{~h})$ on the migration or invasion, respectively of 95D cells. Photomicrographs of migrated/invaded cells are representative of three independent experiments (magnification, $\mathrm{x} 100$; cells are stained with crystal violet). Values are expressed as the mean \pm standard deviation $(\mathrm{n}=3)$. ${ }^{* *} \mathrm{P}<0.01 \mathrm{vs}$. control group. BDMC, bisdemethoxycurcumin.

Western blot analysis. To determine the levels of protein expression, whole-cell lysate extracts of BDMC-treated cells $(0,1,5$ and $10 \mu \mathrm{M}$ for $24 \mathrm{~h}$ and $10 \mu \mathrm{M}$ for $0,6,12$ and $24 \mathrm{~h})$ were prepared and separated by $10 \%$ SDS-PAGE. After electrophoresis, proteins were electroblotted onto a polyvinylidene difluoride membrane (Millipore, Billerica, MA, USA), which was then incubated with the primary antibodies (anti-GAPDH, 1:500; anti-E-cadherin, 1:1,000, anti-vimentin, 1:500). Subsequently, the blots were incubated with the secondary antibody (1:2,000 dilution) for $1 \mathrm{~h}$ at room temperature. Blots were visualized using an enhanced chemiluminescence western blotting system (BeyoECL Plus kit; Beyotime Institute of Biotechnology). The relative amount of protein on the blots was determined by densitometry using Image J software 1.49 (National Institutes of Health, Bethesda, MD, USA).

Statistical analysis. All experiments were performed in triplicate. Values are expressed as the mean \pm standard deviation. The Student's $t$ test was used for paired comparisons, and the one-way analysis of variance test was used for multiple comparisons. SPSS 17.0 (SPSS, Inc., Chicago, IL, USA) was used to analyze the data. $\mathrm{P}<0.05$ was considered to indicate a statistically significant difference.

\section{Results}

BDMC inhibits the proliferation of $95 \mathrm{D}$ cells. In order to investigate whether BDMC inhibits 95D-cell growth, the cells were incubated with increasing concentrations of BDMC $(0$,
$1,5,10,20,40$ or $80 \mu \mathrm{M})$ for $24 \mathrm{~h}$ and subjected to the CCK8 assay. As shown in Fig. 1, the viability of 95D cells treated with BDMC was not significantly reduced compared with that of the untreated control cells at BDMC concentrations of up to $10 \mu \mathrm{M}$, while the proliferation was significantly inhibited by BDMC at $\geq 20 \mu \mathrm{M}$. As treatment with BDMC at concentrations of $0-10 \mu \mathrm{M}$ for $24 \mathrm{~h}$ had no cytotoxic effect on the highly metastatic $95 \mathrm{D}$ cells, this concentration range was used in the subsequent experiments.

BDMC suppresses $95 D$-cell migration and invasion. The present study examined the effects of sub-cytotoxic concentrations of BDMC on the invasive and migratory potential of highly invasive 95D cells. A Boyden chamber assay showed that BDMC $(1-10 \mu \mathrm{M})$ reduced the migratory ability of 95D cells in a dose-dependent manner (Fig. 2A). Similarly, the Transwell invasion assay showed that BDMC (1-10 $\mu \mathrm{M})$ decreased the invasive ability of $95 \mathrm{D}$ cells in a dose-dependent manner (Fig. 2B). These results suggested that BDMC at 1-10 $\mu \mathrm{M}$ significantly suppressed the invasion and migration but not the viability of 95D cells.

Autophagy is involved in BDMC-mediated suppression of $95 D$-cell invasion and migration. At present, autophagy is an important field of cancer research. The effects of autophagy in the tumor are likely to vary depending on the context. Based on the positive regulatory effects of curcumin on autophagy, the present study hypothesized that BDMC suppressed 95D-cell invasion and migration via upregulating autophagy. 
A

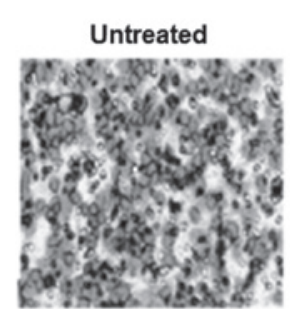

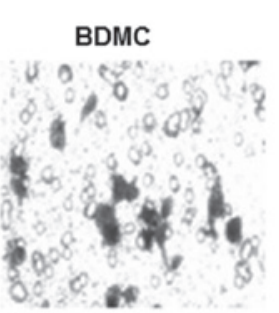

BDMC+ Control siRNA BDMC+ Beclin1 siRNA
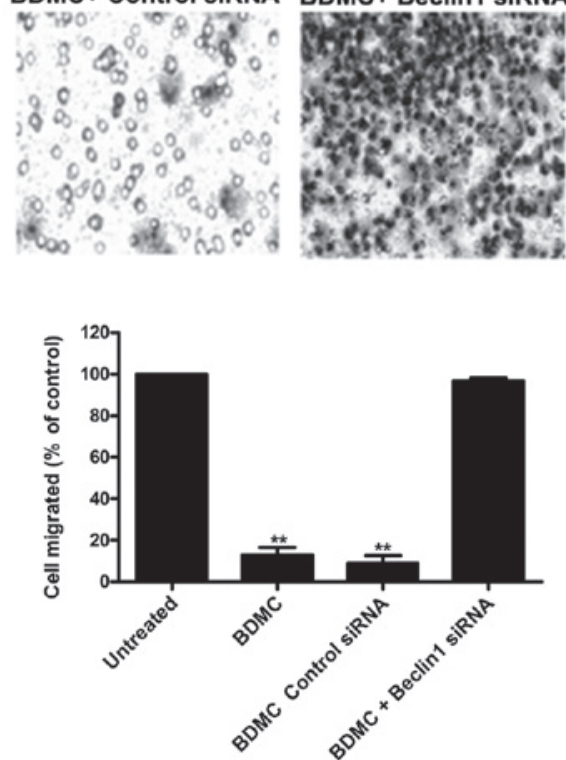

B
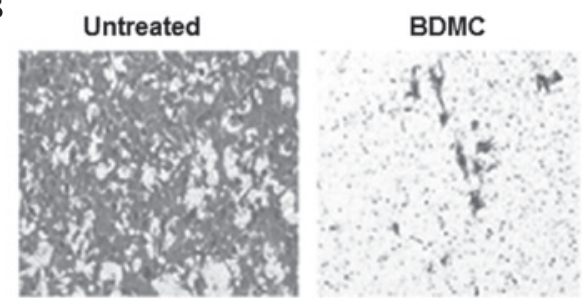

BDMC +Control siRNA BDMC + Beclin1 siRNA
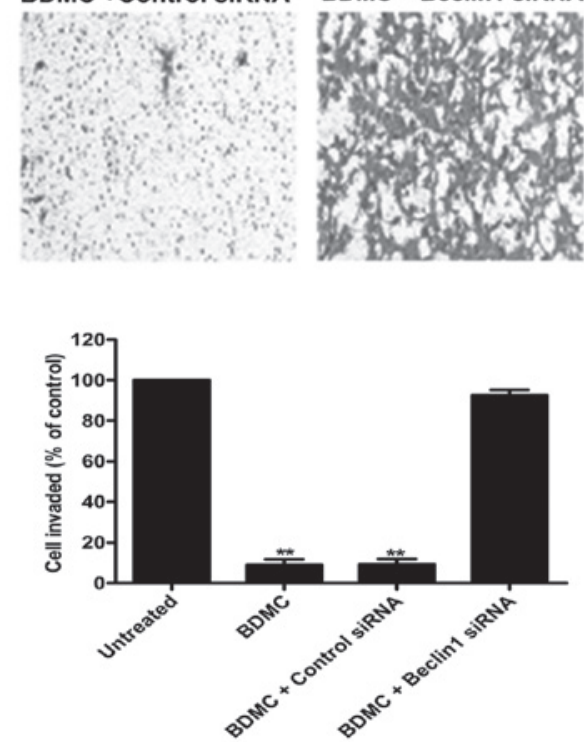

Figure 3. Autophagy is involved in the suppression of 95D-cell invasion and migration by BDMC. Knockdown of Beclin 1 inhibited the suppressive effect of BDMC on (A) 95D-cell migration as assessed by a Boyden chamber assay and (B) 95D-cell invasion as assessed by a Transwell assay in vitro. Beclin 1 siRNA- or control siRNA-expressing 95D cells were treated with BDMC. Photomicrographs of migrated/invaded cells are representative of three independent experiments (magnification, $\mathrm{x} 100$; cells are stained with crystal violet). Values are expressed as the mean \pm standard deviation $(\mathrm{n}=3) .{ }^{* *} \mathrm{P}<0.01 \mathrm{vs}$. untreated group. BDMC, bisdemethoxycurcumin; siRNA, small interfering RNA.
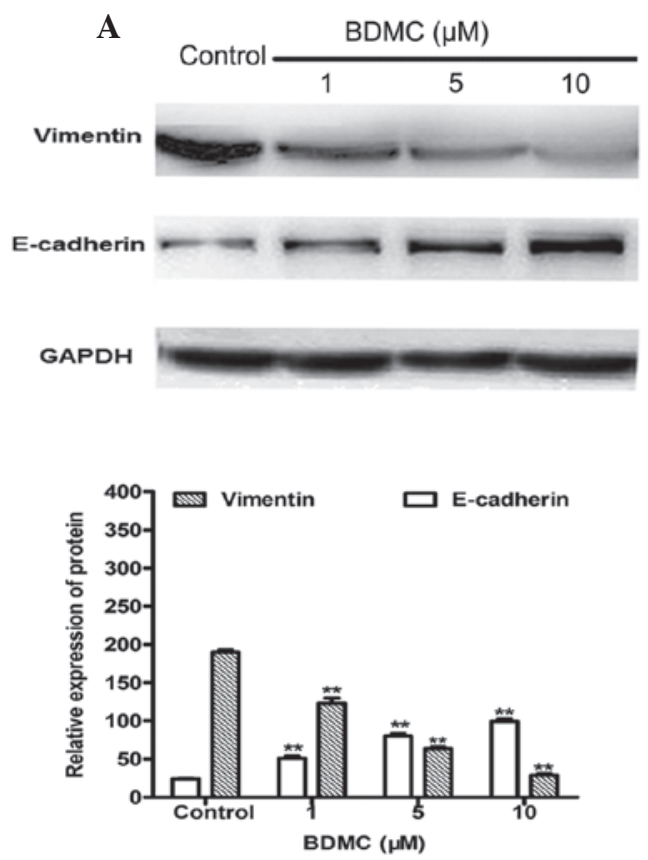

B

\begin{tabular}{llll}
\multicolumn{4}{c}{ BDMC $(10 \mu \mathrm{M})$} \\
\hline $\mathrm{Oh}$ & $6 \mathrm{~h} \quad 12 \mathrm{~h} \quad 24 \mathrm{~h}$
\end{tabular}

E-cadherin

GAPDH

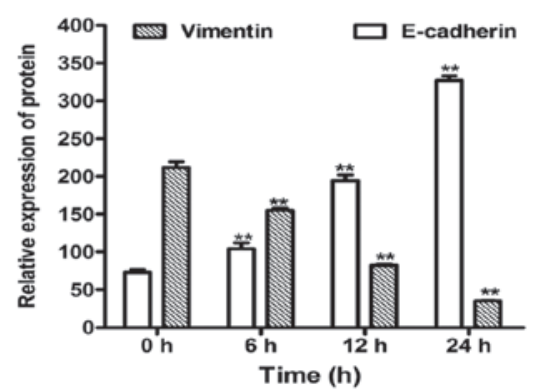

Figure 4. Effects of BDMC on the expression of vimentin and E-cadherin in 95D cells. (A) Cells were treated with the indicated doses of BDMC (1,5 or $10 \mu \mathrm{M})$ for $24 \mathrm{~h}$. (B) Cells were treated with BDMC $(10 \mu \mathrm{M})$ for 6,12 or $24 \mathrm{~h}$. Representative western blots of three independent experiments are shown, and bar graphs display the relative expression levels vimentin and E-cadherin protein levels. GAPDH served as a loading control. Values are expressed as the mean \pm standard deviation $(\mathrm{n}=3) .{ }^{* *} \mathrm{P}<0.01$ vs. untreated group. BDMC, bisdemethoxycurcumin. 
Accordingly, a decrease in autophagy is expected to reverse the effects of BDMC and facilitate invasion and migration. To test this hypothesis, 95D cells were transfected with Beclin 1-siRNA in order to interfere with the formation of autophagosomes (15). Beclin 1 has an important role in the formation of autophagosomes. As shown in (Fig. 3), inhibition of autophagy by Beclin 1 knockdown attenuated the inhibitory effects of BDMC on 95D-cell migration and invasion. This result suggested that BDMC-mediated suppression of 95D-cell invasion and migration proceeds via the autophagic pathway.

BDMC inhibits 95D-cell migration and invasion by modulating the expression of E-cadherin and vimentin. To elucidate the possible underlying mechanisms of anti-metastatic activities of BDMC on 95D cells, its effect on vimentin and E-cadherin expression were assessed by western blot analysis. As shown in Fig. 4, BDMC treatment significantly decreased the expression of vimentin in a dose- and time-dependent manner. Treatment with 1,5 or $10 \mu \mathrm{M}$ BDMC for $24 \mathrm{~h}$ decreased the protein expression of vimentin by $35.2,66.3$ and $84.7 \%$, respectively, compared with that in the control group. In addition, $10 \mu \mathrm{M}$ BDMC treatment for 6,12 and $24 \mathrm{~h}$ decreased vimentin protein expression by $26.7,61.3$ and $83.3 \%$, respectively. Treatment with 1,5 and $10 \mu \mathrm{M}$ BDMC for $24 \mathrm{~h}$ caused a 2.1-, 3.3-, 4.1-fold increase in E-cadherin expression, respectively. BDMC treatment at $10 \mu \mathrm{M}$ for 6, 12 or $24 \mathrm{~h}$ caused 1.4-, 2.6- and 4.5-fold increases in E-cadherin protein expression, respectively. These findings suggested that downregulation of vimentin and upregulation of E-cadherin may be involved in the inhibition of invasion and migration of $95 \mathrm{D}$ cells by BDMC.

\section{Discussion}

Accumulating evidence proved that curcumin has a potent anti-cancer effects in vitro and in vivo on a variety of cancer cell types, including breast cancer (16), prostate cancer (17), melanoma cells (18), ovarian carcinoma (19), pancreatic cancer (20), leukemia (21), head and neck cancer (22) and Burkitt's lymphoma (23). The anti-cancer activities of curcumin are associated with multiple biological pathways involved in tumor growth, oncogene expression, cell cycle regulation, apoptosis, tumorigenesis and metastasis. Although multiple anti-cancer mechanisms of curcumin have been elucidated, the underlying mechanisms by which curcumin inhibits tumor metastasis signaling have remained to be identified. Furthermore, the inhibitory effects of BDMC on the invasion and migration of 95D cells have not yet been investigated. Several studies have shown that curcumin inhibits lung cancer cell invasion and migration (7-9). In agreement with these previous studies, the present study also observed marked inhibitory effects of BDMC on 95D-cell invasion and migration.

A recent study indicated that the induction of the epithelial-to-mesenchymal transition (EMT) has an important role in cancer progression (24). During EMT, epithelial cells lose cell-cell contacts, cell polarity and epithelial markers, particularly E-cadherin, while acquiring mesenchymal markers including vimentin and fibronectin; furthermore, the epithelial cells acquire mesenchymal phenotypes with increased motility and invasiveness (25). Therefore, loss of E-cadherin expression and acquisition of vimentin are the major features of EMT. EMT is considered to be regulated by transcription factors, including Twist, Zeb2 (SIP1), Snail1 and Slug (Snail2) (26,27). E-cadherin is a transmembrane protein mediating cell-cell adhesion between epithelial cells, which has an important role in cell migration and is thus involved in tumor metastasis. Low E-cadherin expression in NSCLC tumors has been reported to be associated with a more aggressive behavior of tumor epithelial cells and with an unfavorable prognosis (28). E-cadherin expression is associated with tumor differentiation, lymph node metastasis and prognosis in patients with NSCLC (29). Vimentin is one of the markers of tumor differentiation and invasion in lung cancer, which predicts the occurrence of metastases in NSCLCs. Vimentin expression was found to be an independent prognostic marker for poor survival in resected NSCLC patients (30). Vimentin overexpression in cancer is correlated with accelerated tumor growth, invasion and poor prognosis $(31,32)$.

In addition, it has been reported that death-effector domain-containing DNA-binding protein activated autophagy and attenuated EMT by inducing the degradation of Snail and Twist in human breast cancer (33). Furthermore, the EMT has been associated with drug resistance and cancer cell metastasis (33). During EMT, the expression of E-cadherin, a transmembrane protein essential for stable adherens junctions, is downregulated, while the expression of the mesenchymal molecules vimentin, fibronectin, and/or N-cadherin is upregulated. In order to investigate the underlying molecular mechanisms of the inhibitory effects of BDMC on 95D-cell migration and invasion, the present study assessed the effects of BDMC on alterations in the protein levels of vimentin and E-cadherin in 95D cells. It was observed that BDMC markedly decreased vimentin expression, while upregulating E-cadherin expression in 95D cells in a dose- and time-dependent manner. These results indicated that BDMC suppressed NSCLC cell invasion and metastasis through the inhibition of vimentin and the promotion of E-cadherin expression.

Autophagy, a strategy of self-adaption, has been regarded as a double-edged sword in tumor metastasis. The role of autophagy in cell migration and invasion has been studied in depth (34). Autophagy is known to exert anti-metastatic effects; however, it also has pro-metastasis activity in certain cases (35). In the present study, autophagy was identified as a target of BDMC, via which its anti-cancer effects, including the inhibition of invasion and migration in NSCLC, were mediated in vitro.

Interfering with the autophagic pathway may affect the migration and invasion of tumor cells. The present study was the first, to the best of our knowledge, to demonstrate that the inhibition of autophagy by knocking down beclin-1 significantly attenuated the inhibitory effects of BDMC on the migration and invasion of $95 \mathrm{D}$ cells, suggesting that autophagy was involved in BDMC-mediated suppression of 95D-cell invasion and migration.

It was therefore hypothesized that BDMC inhibited the invasion and migration of $95 \mathrm{D}$ cells by inducing autophagy, which modulated the EMT process through promotion of the degradation of transcriptional factors, including Twist and Snail. The specific mechanisms require further study.

In conclusion, the present study was the first, to the best of our knowledge, to show that BDMC significantly suppressed 
the invasion and migration of highly metastatic human lung cancer cells through downregulating vimentin and upregulating E-cadherin expression. Furthermore, autophagy was shown to be involved in the anti-tumor effects of BDMC in NSCLC. The mechanisms of action of BDMC are required to be addressed more systematically in further studies.

\section{Acknowledgements}

The authors would like to thank Dr Hu Luo and Miss Qian Qiu, from the department of Respiratory Medicine of Southwest Hospital (Chongqing, China) for their keen interest and help.

\section{References}

1. Siegel R, Naishadham D and Jemal A: Cancer statistics, 2012. CA Cancer J Clin 62: 10-29, 2012.

2. Dempke WC, Suto T and Reck M: Targeted therapies for non-small cell lung cancer. Lung Cancer 67: 257-274, 2010.

3. Kawachi R, Tsukada H, Nakazato Y, Takei H, Furuyashiki G, Koshi-ishi Y and Goya T: Early recurrence after surgical resection in patients with pathological stage I non-small cell lung cancer. Thorac Cardiovasc Surg 57: 472-475, 2009.

4. Ji C, Cao C, Lu S, Kivlin R, Amaral A, Kouttab N, Yang H, Chu W, Bi Z, Di W and Wan Y: Curcumin attenuates EGF-induced AQP3 up-regulation and cell migration in human ovarian cancer cells. Cancer Chemother Pharmacol 62: 857-865, 2008.

5. Zou P, Helson L, Maitra A, Stern ST and McNeil SE: Polymeric curcumin nanoparticle pharmacokinetics and metabolism in bile duct cannulated rats. Mol Pharm 10: 1977-1987, 2013.

6. Sakaue-Sawano A, Kurokawa H, Morimura T, Hanyu A, Hama H, Osawa H, Kashiwagi S, Fukami K, Miyata T, Miyoshi H, et al: Visualizing spatiotemporal dynamics of multicellular cell-cycle progression. Cell 132: 487-498, 2008.

7. Chen HW, Lee JY, Huang JY, Wang CC, Chen WJ, Su SF, Huang CW, Ho CC, Chen JJ, Tsai MF, et al: Curcumin inhibits lung cancer cell invasion and metastasis through the tumor suppressor HLJ1. Cancer Res 68: 7428-7438, 2008.

8. Lin SS, Lai KC, Hsu SC, Yang JS, Kuo CL, Lin JP, Ma YS, Wu CC and Chung JG: Curcumin inhibits the migration and invasion of human A549 lung cancer cells through the inhibition of matrix metalloproteinase-2 and -9 and Vascular Endothelial Growth Factor (VEGF). Cancer Lett 285: 127-133, 2009.

9. Yang CL, Liu YY, Ma YG, Xue YX, Liu DG, Ren Y, Liu XB, $\mathrm{Li} \mathrm{Y}$ and Li Z: Curcumin blocks small cell lung cancer cells migration, invasion, angiogenesis, cell cycle and neoplasia through Janus kinase-STAT3 signalling pathway. PLoS One 7: e37960, 2012.

10. Sandur SK, Pandey MK, Sung B, Ahn KS, Murakami A, Sethi G, Limtrakul P, Badmaev V and Aggarwal BB: Curcumin, demethoxycurcumin, bisdemethoxycurcumin, tetrahydrocurcumin and turmerones differentially regulate anti-inflammatory and anti-proliferative responses through a ROS-independent mechanism. Carcinogenesis 28: 1765-1773, 2007.

11. Yodkeeree S, Chaiwangyen W, Garbisa S and Limtrakul P: Curcumin, demethoxycurcumin and bisdemethoxycurcumin differentially inhibit cancer cell invasion through the down-regulation of MMPs and uPA. J Nutr Biochem 20: 87-95, 2009.

12. Ikeda T, Ishii KA, Saito Y, Miura M, Otagiri A, Kawakami Y, Shimano H, Hara H and Takekoshi K: Inhibition of autophagy enhances sunitinib-induced cytotoxicity in rat pheochromocytoma PC12 cells. J Pharmacol Sci 121: 67-73, 2013.

13. Lagisetty P, Vilekar P, Sahoo K, Anant S and Awasthi V: CLEFMA-an anti-proliferative curcuminoid from structure-activity relationship studies on 3,5-bis (benzylidene) -4-piperidones. Bioorg Med Chem 18: 6109-6120, 2010.

14. Xiao K, Jiang J, Guan C, Dong C, Wang G, Bai L, Sun J, Hu C and Bai C: Curcumin induces autophagy via activating the AMPK signaling pathway in lung adenocarcinoma cells. J Pharmacol Sci 123: 102-109, 2013.

15. Maejima Y, Kyoi S, Zhai P, Liu T, Li H, Ivessa A, Sciarretta S, Del Re DP, Zablocki DK, Hsu CP, et al: Mst1 inhibits autophagy by promoting the interaction between Beclin1 and Bcl-2. Nat Med 19: 1478-1488, 2013.
16. Boonrao M, Yodkeeree S, Ampasavate C, Anuchapreeda S and Limtrakul P: The inhibitory effect of turmeric curcuminoids on matrix metalloproteinase-3 secretion in human invasive breast carcinoma cells. Arch Pharm Res 33: 989-998, 2010.

17. Killian PH, Kronski E, Michalik KM, Barbieri O, Astigiano S, Sommerhoff CP, Pfeffer U, Nerlich AG and Bachmeier BE: Curcumin inhibits prostate cancer metastasis in vivo by targeting the inflammatory cytokines CXCL1 and -2. Carcinogenesis 33: 2507-2519, 2012.

18. Siwak DR, Shishodia S, Aggarwal BB and Kurzrock R: Curcumin-induced antiproliferative and proapoptotic effects in melanoma cells are associated with suppression of IkappaB kinase and nuclear factor kappaB activity and are independent of the B-Raf/mitogen-activated/extracellular signal-regulated protein kinase pathway and the Akt pathway. Cancer 104: 879-890, 2005.

19. Lin YG, Kunnumakkara AB, Nair A, Merritt WM, Han LY, Armaiz-Pena GN, Kamat AA, Spannuth WA, Gershenson DM, Lutgendorf SK, et al: Curcumin inhibits tumor growth and angiogenesis in ovarian carcinoma by targeting the nuclear factor-kappaB pathway. Clin Cancer Res 13: 3423-3430, 2007.

20. Glienke W, Maute L, Wicht J and Bergmann L: Curcumin inhibits constitutive STAT3 phosphorylation in human pancreatic cancer cell lines and downregulation of survivin/BIRC5 gene expression. Cancer Invest 28: 166-171, 2010.

21. Anuchapreeda S, Tima S, Duangrat C and Limtrakul P: Effect of pure curcumin, demethoxycurcumin and bisdemethoxycurcumin on WT1 gene expression in leukemic cell lines. Cancer Chemother Pharmacol 62: 585-594, 2008.

22. Gao W, Chan JY, Wei WI and Wong TS: Anti-cancer effects of curcumin on head and neck cancers. Anticancer Agents Med Chem 12: 1110-1116, 2012.

23. Qiao Q,Jiang Y andLiG:Inhibition of the PI3 K/AKT-NF-kappaB pathway with curcumin enhanced radiation-induced apoptosis in human Burkitt's lymphoma. J Pharmacol Sci 121: 247-256, 2013.

24. Termen S, Tan EJ, Heldin CH and Moustakas A: p53 regulates epithelial-mesenchymal transition induced by transforming growth factor $\beta$. J Cell Physiol 228: 801-813, 2013.

25. Wang $\mathrm{H}$, Zhang $\mathrm{H}$, Tang $\mathrm{L}$, Chen $\mathrm{H}$, Wu C, Zhao M, Yang Y, Chen X and Liu G: Resveratrol inhibits TGF- $\beta 1$-induced epithelial-to-mesenchymal transition and suppresses lung cancer invasion and metastasis. Toxicology 303: 139-146, 2013.

26. Thiery JP: Epithelial-mesenchymal transitions in tumour progression. Nat Rev Cancer 2: 442-454, 2002.

27. Yang J and Weinberg RA: Epithelial-mesenchymal transition: at the crossroads of development and tumor metastasis. Dev Cell 14: 818-829, 2008.

28. Yang YL, Chen MW and Xian L: P rognostic and clinicopathological significance of downregulated E-cadherin expression in patients with non-small cell lung cancer (NSCLC): A metaanalysis. PLoS One 6: e99763, 2014.

29. Liu D, Huang C, Kameyama K, Hayashi E, Yamauchi A, Kobayashi S and Yokomise H: E-cadherin expression associated with differentiation and prognosis in patients with non-small cell lung cancer. Ann Thorac Surg 71: 949-954, 2001.

30. Al-Saad S, Al-Shibli K, Donnem T, Persson M, Bremnes RM and Busund LT: The prognostic impact of NF-kappaB p105, vimentin, E-cadherin and Par6 expression in epithelial and stromal compartment in non-small-cell lung cancer. Br J Cancer 99: 1476-1483, 2008.

31. Satelli A and Li S: Vimentin in cancer and its potential as a molecular target for cancer therapy. Cell Mol Life Sci 68: 3033-3046, 2011.

32. Kokkinos MI, Wafai R, Wong MK, Newgreen DF, Thompson EW and Waltham M: Vimentin and epithelial-mesenchymal transition in human breast cancer-observations in vitro and in vivo. Cells Tissues Organs 185: 191-203, 2007.

33. Lv Q, Wang W, Xue J, Hua F, Mu R, Lin H, Yan J, Lv X, Chen X and Hu ZW: DEDD interacts with PI3KC3 to activate autophagy and attenuate epithelial-mesenchymal transition in human breast cancer. Cancer Res 72: 3238-3250, 2012.

34. Macintosh RL, Timpson P, Thorburn J, Anderson KI, Thorburn A and Ryan KM: Inhibition of autophagy impairs tumor cell invasion in an organotypic model. Cell Cycle 11: 2022-2029, 2012.

35. Zhang Z, Yang L, Xie L, Xu L, Xu D and Liu X: Statins, autophagy and cancer metastasis. Int J Biochem Cell Biol 45: 745-752, 2013. 